\title{
Impairments in microvascular function and skeletal muscle oxygenation in women with gestational diabetes mellitus: links to cardiovascular disease risk factors
}

\author{
Konstantina Dipla $^{1}$ (I) - Areti Triantafyllou ${ }^{2} \cdot$ Iris Grigoriadou $^{1} \cdot$ Evangelia Kintiraki $^{3}$. \\ Georgios A. Triantafyllou ${ }^{2}$ - Pavlos Poulios ${ }^{2}$. Ioannis S. Vrabas ${ }^{1} \cdot$ Andreas Zafeiridis $^{1}$ • \\ Stella Douma ${ }^{2}$ - Dimitrios G. Goulis ${ }^{3}$
}

Received: 15 July 2016/Accepted: 15 September 2016 /Published online: 8 October 2016

(C) Springer-Verlag Berlin Heidelberg 2016

\begin{abstract}
Aims/hypothesis Gestational diabetes mellitus (GDM) is a risk factor for the development of endothelial dysfunction and cardiovascular disease. However, in vivo microvascular endothelial function in GDM has not been investigated. This study aimed to examine, using near-infrared spectroscopy (NIRS), whether: (1) there are differences in microvascular reactivity and skeletal muscle oxygen consumption $\left(\mathrm{m} \dot{V} \mathrm{O}_{2}\right)$ at rest and during exercise between GDM and uncomplicated pregnancies; and (2) there is an association of NIRS indices with macrovascular function and cardiovascular disease risk factors.

Methods Twenty-nine pregnant women (13 with GDM and 16 women with uncomplicated pregnancy, $28 \pm 2$ gestational weeks) underwent arterial stiffness (pulse wave velocity [PWV]) and $24 \mathrm{~h}$ ambulatory BP (24 h BP) evaluation. NIRS continuously monitored, non-invasively, changes in muscle oxygenated and deoxygenated haemoglobin and tissue $\mathrm{O}_{2}$ saturation index (TSI, \%) during arterial occlusion/ reperfusion and intermittent handgrip exercise. $\mathrm{m}^{\mathrm{V}} \mathrm{O}_{2}$ and vascular reactivity indices were calculated.
\end{abstract}

Konstantina Dipla

kdipla@phed-sr.auth.gr

1 Laboratory of Exercise Physiology and Biochemistry, Department of Physical Education and Sports Science at Serres, Aristotle University of Thessaloniki, Ippokratous 22, Agios Ioannis, Serres 62110, Greece

2 Third Department of Internal Medicine, Papageorgiou Hospital, Aristotle University of Thessaloniki, Thessaloniki, Greece

3 Unit of Reproductive Endocrinology, First Department of Obstetrics and Gynaecology, Medical School, Aristotle University of Thessaloniki, Thessaloniki, Greece
Results During occlusion and reperfusion, women with GDM exhibited slower TSI response (occlusion slope: $-0.06 \pm 0.02 \mathrm{vs}$ $-0.10 \pm 0.04$, in GDM and controls, respectively; reperfusion slope: $0.65 \pm 0.26$ vs $1.05 \pm 0.41$, respectively), lower $m \dot{V} \mathrm{O}_{2}$ $\left(1.3 \pm 1.2\right.$ vs $\left.3.8 \pm 2.3 \mu \mathrm{mol} \mathrm{l}^{-1} \mathrm{~min}^{-1}\right)$ and blunted hyperaemia ( $\Delta$ TSI $6.8 \pm 2.9$ vs $9.5 \pm 3.4)$ compared with controls $(p<0.01)$. Despite similar handgrip strength in the GDM and control groups (29.1 \pm 8.1 vs $26.2 \pm 10.4 \mathrm{~kg}$, respectively), during repeated forearm contractions, women with GDM presented a blunted TSI response $(6.5 \pm 3.9$ vs $19.2 \pm 10.9 ; p<0.01)$ and a reduced capacity to maintain the predetermined handgrip (23.4 \pm 2.9 vs $27.4 \pm 3.8 \%, p<0.05)$. NIRS indices correlated with PWV, $24 \mathrm{~h} \mathrm{BP}$ and blood glucose concentration earlier in pregnancy $(r=0.40-0.60 ; p<0.05)$.

Conclusions/interpretation Women with GDM exhibited a characteristic blunted TSI curve, showing alterations in muscle oxygenation and microvascular responsiveness compared with women with uncomplicated pregnancies. These alterations were manifested during exercise and possibly contribute to the reduced exercise tolerance in GDM. NIRS indices correlated with macrovascular indices (arterial stiffness) and $24 \mathrm{~h} \mathrm{BP}$.

Keywords Arterial stiffness · Endothelial function . Exercise $\cdot$ Gestational diabetes mellitus $\cdot$ Microvascular function $\cdot$ Near-infrared spectroscopy $\cdot$ Pulse wave velocity . Skeletal muscle oxygen consumption · Vascular reactivity
Abbreviations
$24 \mathrm{~h} \mathrm{BP} \quad 24 \mathrm{~h}$ ambulatory BP
FMD Flow-mediated dilation
GDM Gestational diabetes mellitus
$\mathrm{HHb} \quad$ Deoxygenated haemoglobin 


$\begin{array}{ll}\mathrm{MVC} & \text { Maximal voluntary contraction } \\ \mathrm{m}_{\dot{V} \mathrm{O}_{2}} & \text { Skeletal muscle oxygen consumption } \\ \mathrm{NIRS} & \text { Near-infrared spectroscopy } \\ \mathrm{O}_{2} \mathrm{Hb} & \text { Oxygenated haemoglobin } \\ \mathrm{PWV} & \text { Pulse wave velocity } \\ \text { tHb } & \text { Total haemoglobin } \\ \mathrm{TSI} & \text { Tissue oxygen saturation index }\end{array}$

\section{Introduction}

Gestational diabetes mellitus (GDM) is a state of glucose intolerance resulting in hyperglycaemia with onset during pregnancy that affects $5-10 \%$ of pregnancies worldwide [1]. Poorly controlled GDM is associated with an increased risk for gestational hypertension, pre-eclampsia [2, 3] and fetal perinatal complications. This metabolic abnormality usually resolves immediately postpartum; however, it has been associated with a higher incidence of type 2 diabetes and increased risk for cardiovascular disease in both the mother and offspring [3-6].

Endothelial dysfunction has been associated with a higher risk for cardiovascular disease in patients with diabetes, showing that the hyperglycaemic environment promotes micro- and macrovascular dysfunction [7, 8]. Although GDM is considered a temporary state as it is alleviated by delivery, some studies have suggested that alterations in endothelial responsiveness occur [9], whereas others have suggested that the duration of exposure to hyperglycaemia is insufficient to induce vascular dysfunction [10,11].

The vast majority of studies examining vascular alterations in GDM have been conducted in rodents or isolated human umbilical cord vessels. These studies have proven insightful in delineating the potential mechanisms underlying GDM pathophysiology; however, they do not provide information on intact circulatory systems. Most in vivo studies in GDM have investigated peripheral conduit artery vasoreactivity using flow-mediated dilation (FMD) $[9,12]$ and only one study has investigated skin microvascular flow [10]. Although FMD provides an insight into the function of large conduit arteries, it does not provide an assessment of downstream hyperaemia within the tissue itself [13]. Moreover, endothelial cells from macro- and microvasculature of different tissues differ in their ability to metabolise substrates involved in vascular tone modulation because of differential expression of genes encoding for membrane transporters for L-arginine and NO synthase [14]. Insulin and adenosine also differentially modulate different cell types because of differences in the abundance of their receptor isoforms in various tissues types $[14,15]$. Thus, information on macrovascular function in GDM does not necessarily apply to the microvascular environment, and functional alterations in one tissue type should not be extrapolated to another. To our knowledge, in vivo microvascular function at the skeletal muscle level in GDM has not been examined.
In this respect, near-infrared spectroscopy (NIRS) allows non-invasive continuous monitoring of functional changes in oxygenated haemoglobin dissociation within the skeletal muscle [13]. Using arterial occlusion and reoxygenation, NIRS technology provides insights into the ability of muscle to extract oxygen from the capillaries and the ability of microvasculature to respond to a stimulus [13, 16-19]. The slope of the tissue $\mathrm{O}_{2}$ saturation curve during reoxygenation provides information on microvascular reactivity that has higher interand intra-day reliability than FMD $[13,16]$.

Studies in non-pregnant individuals with diabetes mellitus have shown that the endothelial dysfunction of capillaries within skeletal muscles can cause an imbalance of oxygen delivery relative to oxygen extraction $[8,20]$, reduced muscle perfusion and mitochondrial dysfunction [20]. In women with GDM, proteomic analysis in abdominal muscles collected at delivery also revealed reduced mitochondrial protein expression and alterations in $\mathrm{Ca}^{2+}$ handling protein content [21]. However, whether impairments in skeletal muscle oxygen consumption $\left(\mathrm{mV}^{\mathrm{V}} \mathrm{O}_{2}\right)$ are present in GDM and are manifested during physiological stress (such as exercise) has not been explored. In addition, it is yet to be examined whether alterations in muscle oxygenation and vascular reactivity contribute not only to exercise intolerance in this population, but to increases in aortic stiffness and BP during pregnancy, encouraging the development of pre-eclampsia. Based on the aforementioned studies, we hypothesised that: (1) alterations in endothelial function and skeletal muscle oxygenation might be present in GDM, reducing vascular reactivity and oxygen utilisation at rest and during exercise; and (2) alterations in microvascular reactivity in women with GDM will be associated with indices of macrovascular (arterial) stiffness and cardiovascular risk factors.

With this in mind, the study aims were to examine whether: (1) differences in skeletal muscle oxygen utilisation and microvascular reactivity exist between GDM and control pregnant women at rest and during intermittent handgrip exercise; and (2) there is an association between muscle oxygenation indices with cardiovascular disease risk factors (arterial stiffness and BP elevations [22, 23]) in GDM and uncomplicated (control) pregnancies. Exploring these variables may assist in the identification of novel risk factors and a better understanding of exercise intolerance in GDM. It may also provide a foundation for future examination of appropriate physical activity interventions to prevent the development of type 2 diabetes in the postpartum years.

\section{Methods}

Participants Twenty-nine pregnant women (13 women with GDM and 16 age- and parity-matched women), $28 \pm 2$ 
gestational weeks, were recruited from the 1st ObstetricsGynaecology Clinic of Papageorgiou Hospital, Aristotle University, Thessaloniki, Greece, during 2015, for this casecontrol study. The selection criteria for the GDM and control groups were: (1) normotensive women $(\mathrm{BP}<140 / 90 \mathrm{mmHg}$ ) and no proteinuria ( $24 \mathrm{~h}$ urine albumin $\leq 300 \mathrm{mg}$ ); and (2) otherwise healthy (i.e. no pre-existing diabetes, no known cardiovascular or pulmonary disease). In accordance with the American Diabetes Association [1], the diagnosis of GDM was made when at least one of the following plasma glucose values was exceeded: fasting glucose $\geq 5.1 \mathrm{mmol} / 1 ; 1 \mathrm{~h}$ plasma glucose $\geq 10.0 \mathrm{mmol} / \mathrm{l}$; or $2 \mathrm{~h}$ plasma glucose $\geq 8.5 \mathrm{mmol} / \mathrm{l}$ during the OGTT. The one-step $75 \mathrm{~g}$ OGTT was performed in the morning after an overnight fast of at least $8 \mathrm{~h}$ [1]. Immediately after diagnosis, women in the GDM group followed medical nutritional therapy and were advised to increase physical activity. If normoglycaemia was not achieved after 7-10 days with lifestyle changes, insulin treatment was initiated [1]. This study was approved by the institutional review board committee and conducted in accordance with the Helsinki Declaration (1975, 1983 revision). Prior to enrolment, each participant signed the written informed consent form. Participants were asked to follow their normal diet and have sufficient rest the night before the study. None of the participants was highly active or participated in organised training sessions during pregnancy or the year before pregnancy.

Testing procedures and instrumentation Participants were recruited at the time of the OGTT (at the 24-25th gestational week, on average). During this first visit, personal, obstetric and family histories were obtained and women in the GDM group initiated medical nutritional therapy and were advised to increase their physical activity. If they failed to control blood glucose levels to within the optimal values in 7-10 days, pharmaceutical treatment was initiated. Four weeks later (second visit at $28 \pm 2$ gestational weeks, on average), participants reported to the clinic for the implementation of the NIRS protocol (occlusion and exercise), vascular assessment and $24 \mathrm{~h}$ ambulatory BP ( $24 \mathrm{~h} \mathrm{BP})$ monitoring. During this second visit, six patients with GDM were on insulin treatment (one injection of long-acting insulin per day) and seven patients were receiving medical nutritional therapy. Participants were oriented and familiarised with experimental procedures during the visit. Physical characteristic assessment and blood testing were performed. Resting BP was assessed using two measures in the sitting position (digital Omron, Kyoto, Japan). Arterial stiffness was estimated by pulse wave velocity (PWV; Sphygmocor, AtCor Medical, West Ryde, NSW, Australia) according to a standard protocol [24]. Next, the NIRS device (Portamon, Artinis Medical Systems, Elst, the Netherlands) was placed at the participant's forearm to non-invasively monitor changes in muscle oxygenation [18, 25]. The NIRS device assesses relative changes from baseline for oxygenated haemoglobin $\left(\mathrm{O}_{2} \mathrm{Hb}\right)$ and deoxygenated haemoglobin $(\mathrm{HHb})$, and calculates total haemoglobin $(\mathrm{tHb})$ and the tissue oxygen saturation index (TSI) as absolute parameter for muscle oxygenation [26]. During NIRS measurements, the participant remained in the resting position for approximately $20 \mathrm{~min}$ and arterial occlusion followed. The cuff was rapidly inflated to suprasystolic levels (i.e. $250 \mathrm{mmHg}$ ) for $5 \mathrm{~min}$, in order to obstruct blood flow to the forearm muscles and measure the maximal capacity for $\mathrm{O}_{2}$ extraction by skeletal myocytes [13, 16, 19]. Stable blood flow/volume was verified by $\mathrm{tHb}$. Participants reached a steady-state TSI at the end of the occlusion. The cuff was then rapidly deflated and reperfusion indices were recorded.

After a subsequent $10 \mathrm{~min}$ rest, the participant performed three maximal isometric handgrip contractions with their dominant hand using a digital dynamometer (MP150, Biopac, Goleta, CA, USA) with a 90 s interval between trials. The highest of the three readings was considered the maximal voluntary contraction (MVC). Next, the participant performed a 3 min submaximal intermittent handgrip exercise test (4 s exercise at $35 \% \mathrm{MVC}$ with a $3 \mathrm{~s}$ rest), during which she had visual feedback to maintain force output to the predetermined percentage of her MVC. Beat-by-beat BP was monitored throughout the protocol (Finapres, Finapres Medical Systems, Amsterdam, the Netherlands). Adipose tissue thickness (Harpenden skinfold calliper, British Indicators, Burgess Hill, UK) at the portamon site (mid-distance between light source and detector) was measured.

$\mathrm{m}^{\mathrm{V}} \mathrm{O}_{2}\left(\mu \mathrm{mol} \mathrm{l}^{-1} \mathrm{~min}^{-1}\right)$ was calculated from the upsloping $\mathrm{HHb}$ with stable $\mathrm{tHb}$. Obstruction of both inflow and outflow results in a static compartment where the increase in $\mathrm{HHb}$ is directly related to consumption. The TSI slope following cuff release (reperfusion) was used as an index of the reactivity of vessels to accommodate the increase in blood flow [13]. The magnitude of hyperaemic response was calculated as the difference between peak TSI during reperfusion and baseline TSI $(\Delta \mathrm{TSI} \%)$. During exercise, TSI and $\mathrm{HHb}$ indices were used as indices of muscle oxygenation and microvascular $\mathrm{O}_{2}$ extraction [27, 28]. Handgrip and NIRS oxygenation measurements have been shown to be valid and highly reliable [16, 18, 29-31].

For $24 \mathrm{~h}$ BP monitoring, participants were equipped with portable devices (Spacelabs 90207; Spacelabs Healthcare, Redmond, WA, USA), programmed to record BP at $20 \mathrm{~min}$ intervals during a typical work day and $30 \mathrm{~min}$ intervals during the subsequent night.

Statistical analyses Data are reported as mean \pm SD. For statistical analysis, the NIRS-derived variables were averaged over the testing periods (Oxysoft, Artinis Medical Systems, Elst, the Netherlands). Minimal and maximal values, and their difference (i.e. magnitude of change), were calculated for NIRS variables. A regression line $(y=\alpha+b x)$ was applied to 
the occlusion and reperfusion data (where $b$ is the slope and $a$ the intercept). Differences between groups were assessed by Student's $t$ tests for independent samples. Two-way ANOVA with repeated measures was used to assess the effect of 'group' and 'time' (baseline, occlusion and reperfusion), followed by Tukey post-hoc in case of significant group $\times$ time interaction (Statistica 7.0, StatSoft, Tulsa, OK, USA). Pearson correlation $(r)$ was used to measure the relationship between NIRS variables, macrovascular function and BP.

\section{Results}

Women's characteristics The participants' characteristics are presented in Table 1. Participants in the GDM and control groups were of similar chronological age. At the first visit (at the time of GDM diagnosis), significant differences $(p<0.05)$ between groups were observed, as expected, in fasting blood glucose, blood glucose measurements during
OGTT, body weight and BMI. During the second visit, women in both groups were of similar gestational age $(28.1 \pm 2.3$ and $28.0 \pm 2.5$ weeks, GDM and control group, respectively), with no significant differences between groups in haematocrit, haemoglobin, total cholesterol and blood glucose (with GDM patients under treatment). No differences between groups were observed in: (1) clinic and $24 \mathrm{~h}$ BP measurements; (2) PWV; and (3) forearm skinfold at the site of measurement $(5.15 \pm 0.87$ and $5.06 \pm 0.96 \mathrm{~mm}, \mathrm{GDM}$ and control group, respectively).

Brachial artery occlusion and reperfusion The TSI responses from the GDM and control groups during arterial occlusion, reperfusion and hyperaemia are presented in Fig. 1 (curves constructed by averaging TSI data per group). The TSI was not significantly different between groups at baseline $(69.6 \pm 4.0$ vs $66.7 \pm 3.0 \%$ in GDM and control groups, respectively) and declined $(p<0.001)$ in both groups during occlusion. However, women with GDM exhibited a
Table 1 Participant characteristics and study outcomes

\begin{tabular}{|c|c|c|c|}
\hline Variable & GDM & Controls & $p$ value \\
\hline Age (years) & $34.4 \pm 3.6$ & $35.0 \pm 3.5$ & 0.631 \\
\hline \multicolumn{4}{|l|}{ Visit 1 (at GDM diagnosis) } \\
\hline Fasting blood glucose (mmol/l) & $5.4 \pm 0.8$ & $4.6 \pm 0.5$ & 0.030 \\
\hline \multicolumn{4}{|l|}{ OGTT } \\
\hline Glucose at baseline $(\mathrm{mmol} / \mathrm{l})$ & $5.4 \pm 0.8$ & $4.5 \pm 0.3$ & 0.001 \\
\hline Glucose at $60 \mathrm{~min}(\mathrm{mmol} / \mathrm{l})$ & $10.3 \pm 2.6$ & $7.4 \pm 1.5$ & 0.001 \\
\hline Glucose at $120 \mathrm{~min}(\mathrm{mmol} / \mathrm{l})$ & $7.4 \pm 1.8$ & $5.7 \pm 1.5$ & 0.010 \\
\hline \multicolumn{4}{|l|}{ Visit 2 (4 weeks after GDM diagnosis) } \\
\hline Body weight $(\mathrm{kg})$ & $91.9 \pm 20.3$ & $73.4 \pm 15.7$ & 0.009 \\
\hline $\operatorname{BMI}\left(\mathrm{kg} / \mathrm{m}^{2}\right)$ & $33.5 \pm 6.3$ & $26.9 \pm 5.6$ & 0.005 \\
\hline Fasting blood glucose $(\mathrm{mmol} / \mathrm{l})$ & $4.4 \pm 0.8$ & $4.4 \pm 0.7$ & 0.895 \\
\hline Total cholesterol $(\mathrm{mmol} / \mathrm{l})$ & $6.8 \pm 1.0$ & $6.7 \pm 1.1$ & 0.848 \\
\hline HDL-cholesterol (mmol/l) & $1.6 \pm 0.3$ & $1.8 \pm 0.3$ & 0.196 \\
\hline LDL-cholesterol (mmol/l) & $4.0 \pm 0.9$ & $4.0 \pm 1.1$ & 0.981 \\
\hline Haematocrit $(\%)$ & $36.5 \pm 2.5$ & $36.9 \pm 1.6$ & 0.570 \\
\hline Haemoglobin (g/l) & $112 \pm 7$ & $112 \pm 8$ & 0.924 \\
\hline \multicolumn{4}{|l|}{ Clinic BP (mmHg) } \\
\hline Systolic (mmHg) & $113.3 \pm 11.9$ & $107.5 \pm 11.2$ & 0.308 \\
\hline Diastolic (mmHg) & $74.2 \pm 10.4$ & $70.1 \pm 5.6$ & 0.335 \\
\hline Heart rate (bpm) & $90.8 \pm 10.0$ & $83.2 \pm 17.8$ & 0.286 \\
\hline \multicolumn{4}{|l|}{ Ambulatory BP (mmHg) } \\
\hline Systolic (mmHg) & $112.1 \pm 8.5$ & $113.9 \pm 7.5$ & 0.554 \\
\hline Diastolic (mmHg) & $67.3 \pm 6.0$ & $66.8 \pm 5.1$ & 0.819 \\
\hline Heart rate (bpm) & $86.9 \pm 5.2$ & $89.6 \pm 10.2$ & 0.374 \\
\hline $\mathrm{PWV}(\mathrm{m} / \mathrm{s})$ & $5.51 \pm 0.89$ & $5.25 \pm 0.72$ & 0.397 \\
\hline RPI & $0.880 \pm 0.218$ & $0.927 \pm 0.428$ & 0.745 \\
\hline LPI & $0.885 \pm 0.274$ & $0.822 \pm 0.199$ & 0.520 \\
\hline
\end{tabular}

Data are given as mean $\pm \mathrm{SD}$

LPI, left uterine pulsatile index; RPI, right uterine pulsatile index 
Fig. 1 TSI curves during arterial occlusion and reperfusion constructed from averaging data in (a) GDM and (b) control groups

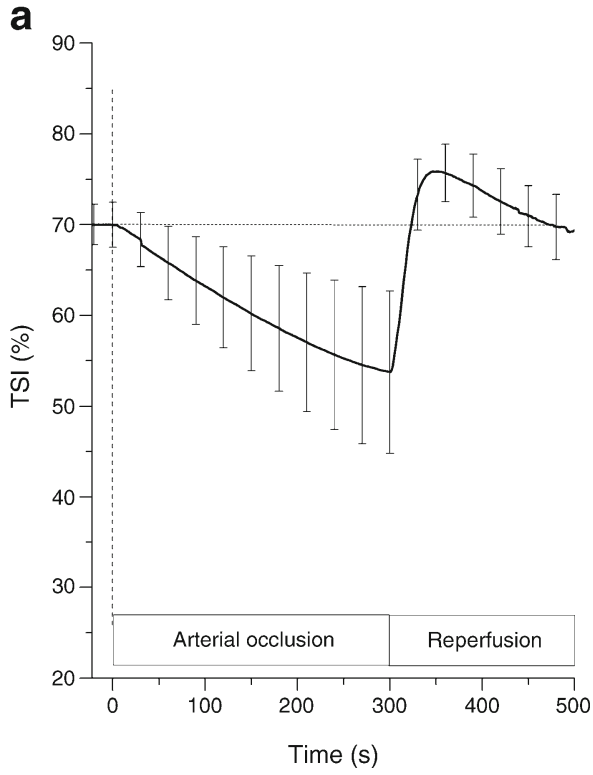

markedly blunted $(p<0.001)$ TSI response during this ischaemic period compared with the control counterparts (TSI occlusion magnitude $-16.05 \pm 6.71$ vs $-25.90 \pm 9.81$ for GDM vs controls, respectively; Fig. 2b) and a slower rate of deoxygenation (TSI occlusion slope: $-0.06 \pm 0.02$ vs $-0.10 \pm 0.04$, respectively, $p<0.01$; Fig. $2 \mathrm{a})$. $m \dot{\mathrm{V}} \mathrm{O}_{2}(\mathrm{HHb})$ was lower in GDM than controls $\left(1.3 \pm 1.2\right.$ vs $3.8 \pm 2.3 \mu \mathrm{mol} \mathrm{l}^{-1} \mathrm{~min}^{-1}$, respectively, $p=0.001$; Fig. 2 c).

During reperfusion, TSI rapidly increased $(p<0.001)$ and exceeded baseline levels in both groups (Fig. 1); however, women with GDM presented a slower reoxygenation rate than controls (TSI reperfusion slope: $0.65 \pm 0.26$ vs $1.05 \pm 0.41$, respectively; $p<0.01$; Fig. 2d), suggesting a reduced microvascular reactivity in the former group. The magnitudes of TSI (\%) reperfusion $(22.9 \pm 9.3$ vs $35.4 \pm 11.9$, respectively, $p<0.01$; Fig. $2 \mathrm{e}$ ) and hyperaemia ( $\Delta$ TSI: $6.8 \pm 2.9$ vs 9.5 $\pm 3.4, p<0.05$; Fig. 2f) were smaller in GDM than controls.

Intermittent exercise Representative data for tissue oxygenation during handgrip in a GDM and a control participant are presented in Fig. 3. During exercise, women with GDM exhibited a lower $(p<0.05)$ magnitude of TSI decline and $\mathrm{HHb}$
Fig. 2 TSI during occlusion in GDM and control groups: slope

(a), magnitude (b) and $\mathrm{m} \dot{\mathrm{V}}{ }_{2}$ (c). TSI during reperfusion: slope (d), magnitude (e) and hyperaemic response (f). ${ }^{* *} p<0.01$ vs GDM group
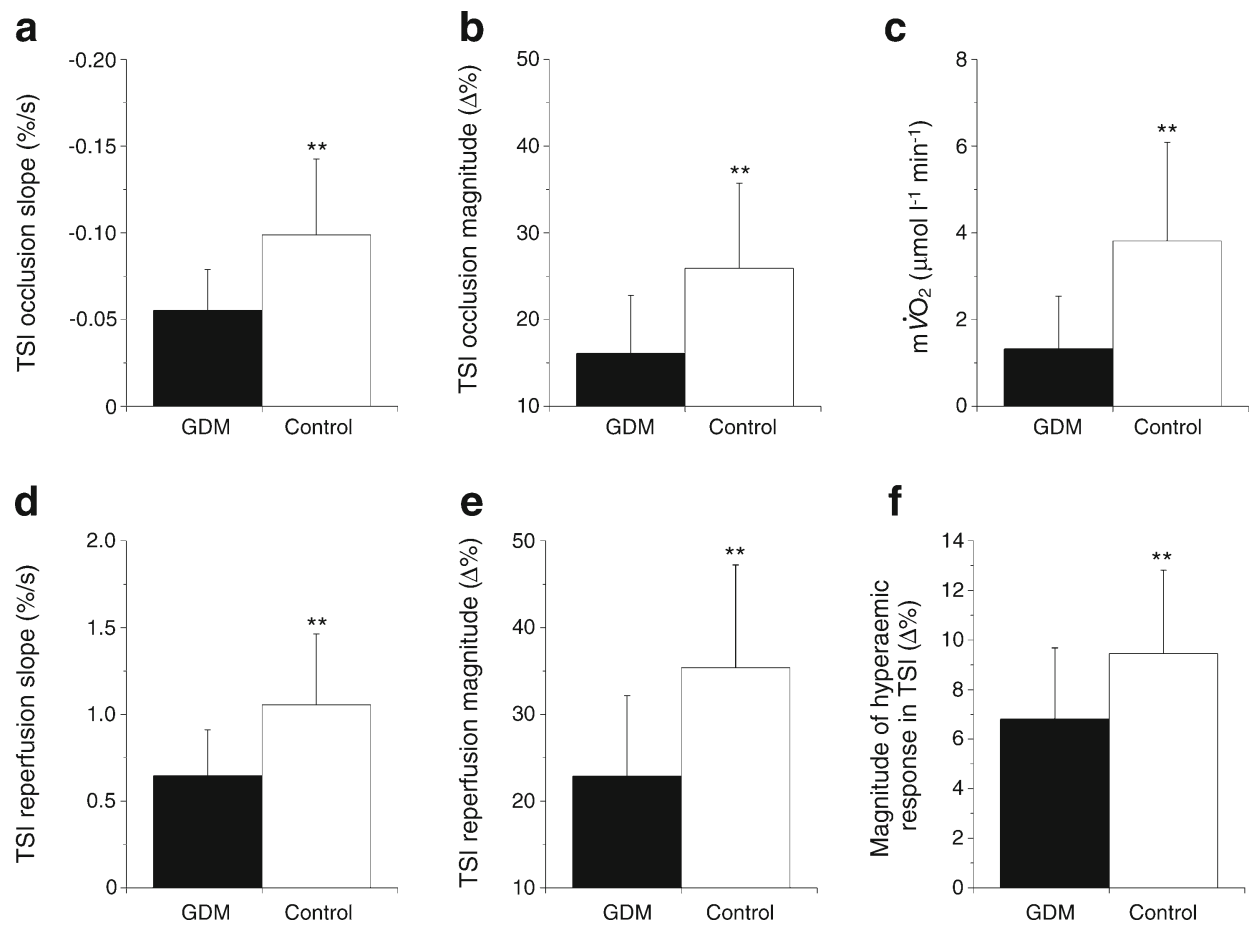
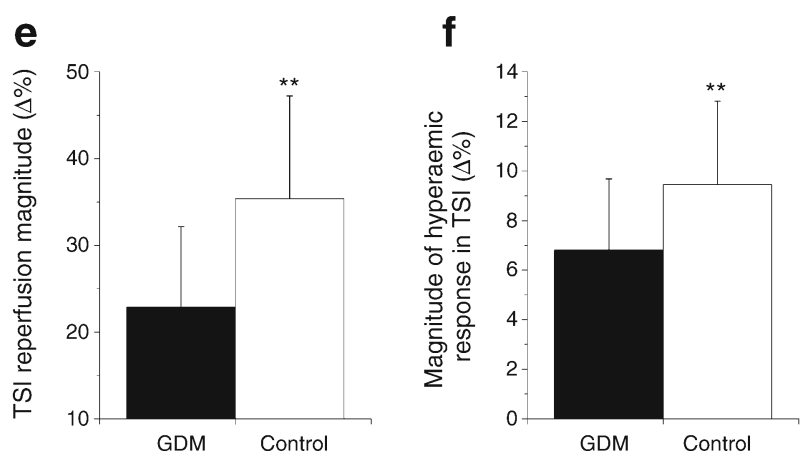
Fig. 3 Representative data for TSI during the 3 min intermittent handgrip exercise in (a) a woman with GDM and (b) a control participant. Grey line, $\mathrm{HHb}$; black line, $\mathrm{O}_{2} \mathrm{Hb}$; and dotted line, $\mathrm{tHb}$

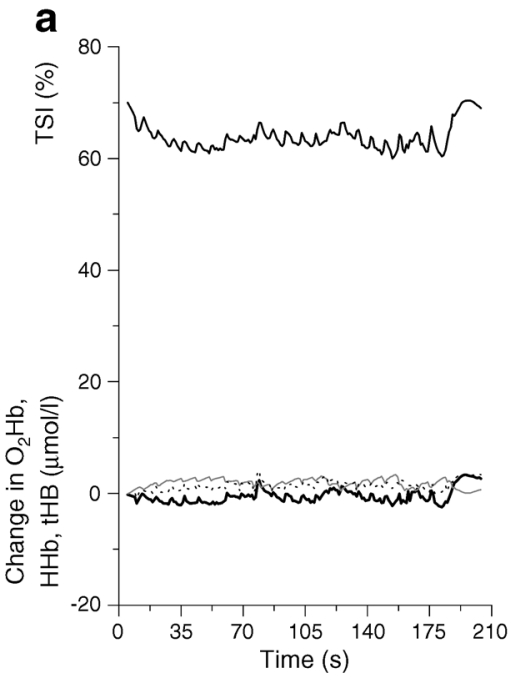

rise (Fig. 4) and a lower $(p<0.05)$ peak response in $\mathrm{tHb}$. Although no differences were observed in MVC between groups $(29.1 \pm 8.1 \mathrm{vs} 26.2 \pm 10.4 \mathrm{~kg}$ in GDM and controls, respectively), during the $3 \mathrm{~min}$ exercise session, women with GDM were not able to maintain the same average MVC percentage as their control counterparts $(23.4 \pm 2.9 \%$ vs 27.4 $\pm 3.8 \%, p<0.05)$.

Relationship between muscle oxygenation, arterial stiffness and cardiovascular risk factors Significant correlations $(r=0.40-0.60)$ were found between muscle oxygenation variables (during occlusion) with arterial stiffness (assessed by PWV) and $24 \mathrm{~h}$ ambulatory systolic and diastolic BP. The TSI occlusion slope and $\mathrm{m} \dot{V} \mathrm{O}_{2}(\mathrm{HHb})$ significantly correlated with PWV $(r=0.56$ and $r=-0.60$, respectively, $p<0.01$; Fig. 5a, b). PWV was correlated with muscle deoxygenation ( $\mathrm{HHb}$ magnitude) during exercise $(r=0.60, p<0.001)$. No significant correlation was observed between tissue oxygenation and participants' age.

Significant correlations $(p<0.05)$ were also observed between NIRS variables (tested on average at $28 \pm 2$ weeks) and blood glucose levels during OGTT (assessed 4 weeks earlier, before women started treatment). More specifically, the TSI occlusion slope was correlated with baseline blood glucose during OGTT ( $r=0.502, p<0.01$; Fig. 5c); and TSI reperfusion slope correlated $(p<0.01)$ with baseline $(r=-0.577)$ and $60 \mathrm{~min}(r=-0.390)$ blood glucose during OGTT (Fig. 5d). No significant correlation was observed between oxygenation variables and blood glucose concentration at the time of NIRS measurement when women were treated for hyperglycaemia.

Comparisons of BMI-matched groups In a subset of data, we compared 16 BMI pair-matched pregnant women (BMI: $30.9 \pm 5.7$ vs $29.9 \pm 5.9 \mathrm{~kg} / \mathrm{m}^{2}$, GDM $[n=8]$ vs controls $[n=8]$, respectively; $p=0.8)$. The statistical analysis revealed similar results to those for the whole cohort (TSI occlusion magnitude: $-15.89 \pm 4.4$ vs $-24.62 \pm 9.06, p<0.05$; TSI occlusion slope: $-0.053 \pm 0.016$ vs $-0.092 \pm 0.037, p<0.01$; $\mathrm{m}_{\dot{V}} \mathrm{O}_{2}(\mathrm{HHb}): 1.3 \pm 0.6$ vs $2.9 \pm 1.9 \mu \mathrm{mol} \mathrm{l}^{-1} \mathrm{~min}^{-1}, p<0.05$, in GDM and controls, respectively).

\section{Discussion}

This study examined, for the first time: (1) microvascular reactivity and skeletal muscle oxygenation in GDM and control pregnant women at rest and during intermittent handgrip exercise using NIRS; and (2) the associations of NIRS indices with aortic stiffness and cardiovascular risk factors. Women with GDM exhibited a characteristic blunted TSI curve during
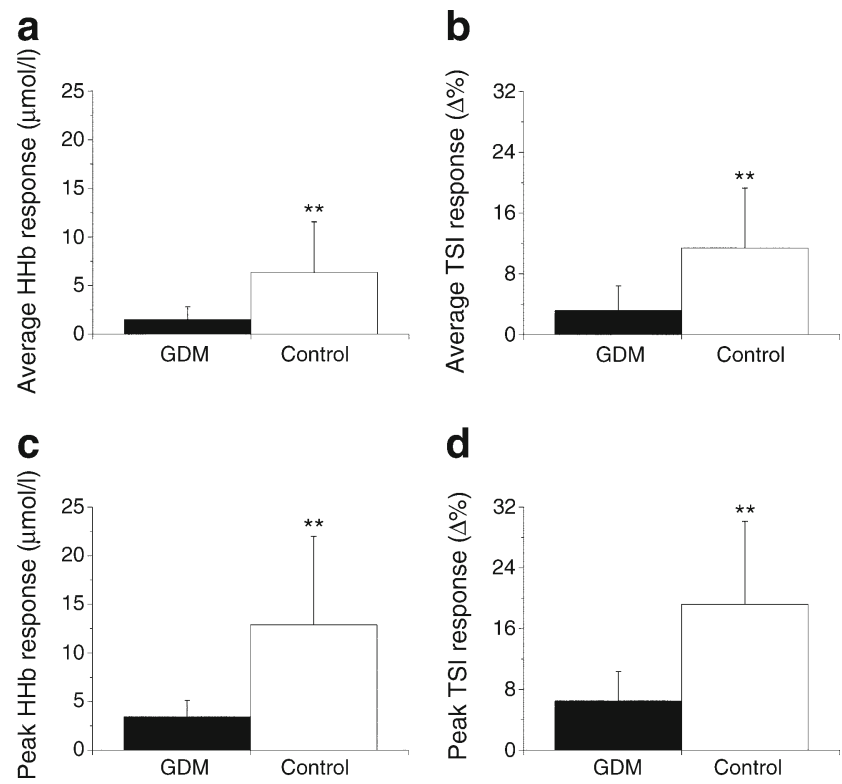

Fig. 4 Average $(\mathbf{a}, \mathbf{b})$ and peak $(\mathbf{c}, \mathbf{d})$ responses in skeletal muscle oxygenation during handgrip in GDM and control groups. $p<0.01$ vs GDM 
Fig. 5 Correlation of PWV with TSI occlusion slope (a) and $\mathrm{m} \dot{V} \mathrm{O}_{2}$ (b); correlation of blood glucose at GDM diagnosis (tested at 24-25th gestational week) with TSI occlusion slope (c) and TSI reperfusion slope (d) (tested on average at the 28th gestational week)
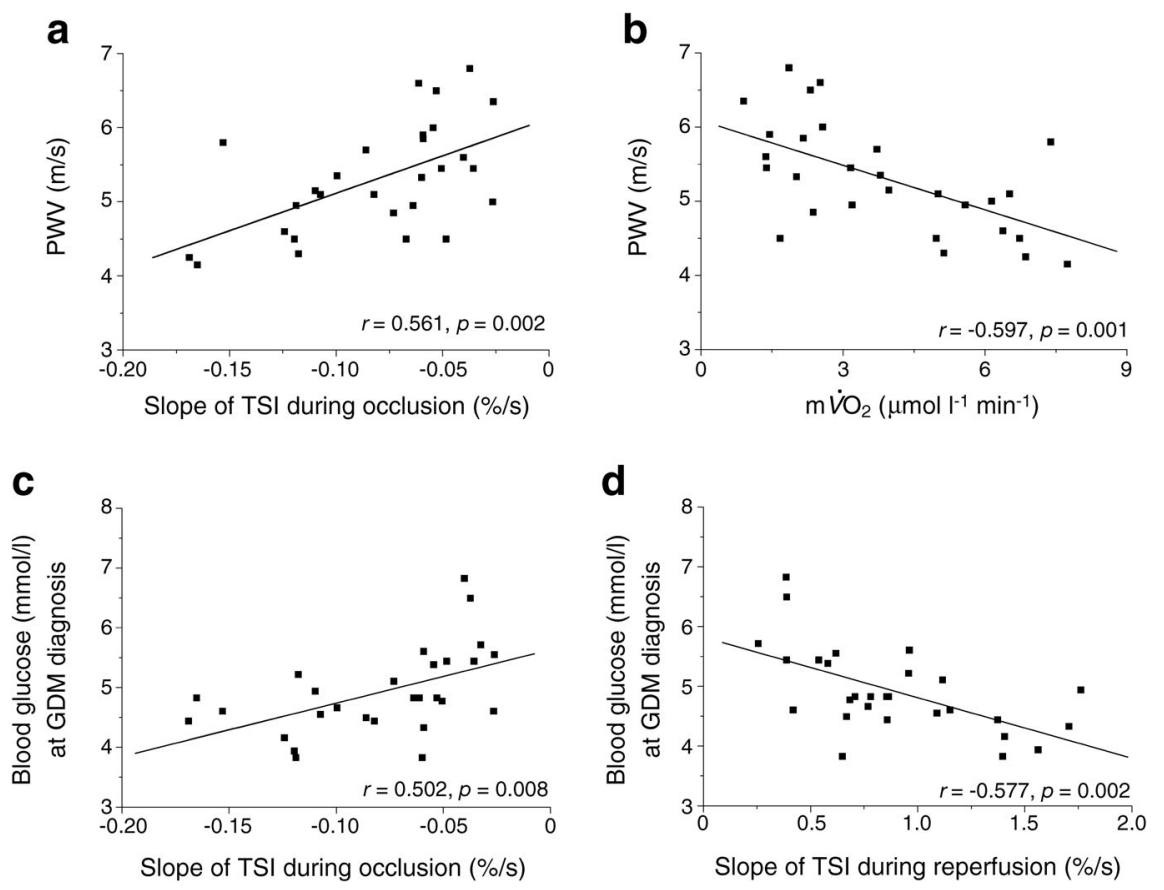

occlusion because of alterations in skeletal muscle oxygenation capacity and microvascular reactivity. These dysfunctions were also manifested during submaximal exercise and were significantly correlated with macrovascular indices and cardiovascular disease risk factors. Although none of the pregnant women had clinical signs of gestational hypertension or impaired aortic stiffness, the significant correlation of muscle reoxygenation slope with $\mathrm{PWV}$ and $24 \mathrm{~h}$ BP measurements imply a link between microvascular reactivity and early macrovascular alterations. Notably, significant correlations were observed between muscle oxygenation indices (assessed on average at 28 weeks) and first visit blood glucose levels (before women with GDM initiated treatment). The microvascular and oxygenation differences between groups persisted when adjusting for BMI differences. The blunted muscle oxygenation during exercise in GDM was reflected in their reduced ability to maintain the required MVC percentage during the 3 min exercise.

Assessment of vascular endothelial integrity in humans can be performed by means of biochemical markers or by functional tests, such as the FMD test $[9,12,32]$. The cost and validity of some circulating biomarkers has, so far, hindered their implementation as routine prenatal screening tests for endothelial dysfunction [33]. The FMD test, on the other hand, is a low-cost test that uses ultrasound to detect large artery vasoreactivity. Although significant correlations of FMD- and NIRS-derived measures during occlusion/reperfusion have been recently shown in non-pregnant adults [13], the FMD test: (1) primarily focuses on changes in conduit artery dilation from a baseline to a post-occlusion value expressed as per cent dilation, which neglects dynamic adjustments of the response; and (2) does not assess microvascular function within the tissue itself $[13,14]$. There are currently some reports on FMD and macrovascular function in GDM [9]; however, cellular mechanisms of the macrovascular endothelium should not necessarily be extrapolated to the microvascular endothelium [14]. The macrovascular endothelium is less reactive to growth factors (i.e. placental growth factor), has lower homeobox gene expression and responds differently to hormonal stimuli and vasoactive molecules (insulin, NO, adenosine) than the microvascular endothelium [14]. Although microvascular abnormalities may precede the clinical manifestations of diabetic microvascular dysfunction, in vivo microvascular function has been underinvestigated in GDM. The NIRS technology used in this study allows noninvasive determination of the magnitude and dynamic adjustment of microvascular responses within the skeletal muscle in humans and provides reliable continuous recordings of local skeletal muscle oxygenation at rest and during exercise that is not possible with the previously used methods [13].

Women in the GDM group exhibited a reduced capacity for $\mathrm{O}_{2}$ extraction at the skeletal muscle level, as shown by the slower slope during occlusion and lower skeletal $m \dot{V} \mathrm{O}_{2}$ vs controls, implying reduced oxidative capacity and mitochondrial function [31]. Our findings agree with in vitro and in situ studies showing reduced mitochondrial capacity in cells and isolated mitochondria in diabetes type 2 or GDM [20, 21], and also extend the knowledge of mitochondrial function under in vivo conditions.

The GDM group also exhibited a slow reoxygenation rate, suggesting alterations in the balance between $\mathrm{O}_{2}$ delivery and $\mathrm{O}_{2}$ extraction. During reperfusion, there is a rapid increase in forearm blood flow into the muscles due to, at least partly, 
increased conductance mediated by the release of local vasodilators [34]. Therefore, the sluggish and delayed response in GDM participants probably reflects a reduced release of vasodilators from the vascular endothelium, an inability of small vessels (i.e. terminal arterioles) to dilate in response to stimuli and/or a capillary rarefaction. In accordance, studies in arteries from mice with previous GDM or human placental cells showed a reduced endothelium-dependent vasodilatation or larger contraction than normal vessels when exposed to post-hypoxic reoxygenation or hydrogen peroxide $[35,36]$. Possible mechanisms proposed for cells from mouse models of GDM and cultured cells from individuals with type 2 diabetes include increased superoxide production caused by mitochondrial uncoupling, which decreases NO bioavailability by peroxynitrite formation [35], impairs $\mathrm{K}^{+}$channels and induces nitrosylation of proteins and DNA damage [35, 37].

The blunted hyperaemic response in the GDM group gives an early indication of microvascular dysfunction [19]. Notably, although none of the GDM participants presented clinical signs of macrovascular dysfunction or gestational hypertension, the TSI responses were significantly correlated with PWV, a gold standard measure of arterial (macrovascular) stiffness and a prognostic factor for cardiovascular disease [38]. The microvascular alterations observed in the GDM group can hinder the fundamental pregnancy adaptation for vasodilatation $[9,39]$ and this is of particular importance during exercise, as it can limit $\mathrm{O}_{2}$ delivery to active muscles and induce premature fatigue in a pregnant woman with diabetes. Indeed, the reduced capacity for muscle oxygenation during occlusion in GDM was reflected in the blunted tissue oxygenation during the handgrip test. Participants in both groups exhibited similar MVCs (i.e. similar strength levels). However, during repeated contractions the GDM group exhibited a lower magnitude of TSI decline and an inability to maintain the required MVC percentage. These data suggest that peripheral impairments, not only central limitations, are important contributors to low physical fitness in women with GDM.

Another important factor that can limit the vasodilatory capacity of the exercising muscle is an exaggerated sympathetic tone. Obesity, a central attribute to GDM [5], can cause an increased sympathetic stimulation [40]. The involvement of the autonomic nervous system in microvascular dysfunction in GDM requires further study. Nevertheless, as increased BMI is a characteristic feature of GDM [41], BMI-matched individuals were compared in a subset of data. The delayed and blunted TSI responses during occlusion and exercise were still evident in GDM vs BMI-matched control women.

There is controversy in the literature regarding whether hyperglycaemia and insulin resistance causes endothelial dysfunction or whether endothelial dysfunction already exists prior to GDM diagnosis and is unmasked by pregnancy $[35,42]$. Women in our GDM group were diagnosed and treated early for their hyperglycaemia; however, they still presented signs of microvascular endothelial dysfunction. 'Hyperglycaemic memory', a mechanism described in animals and humans with type 2 diabetes [8], cannot be excluded, as significant correlations were found between muscle oxygenation variables (measured on average at 28 weeks) and glucose levels at the time of GDM diagnosis (24-25th week). Possible mechanisms such as the generation of irreversible AGEs or oxidative stress, which have been implicated in the induction of a hyperglycaemic memory and long-term arterial stiffness in individuals with impaired glucose tolerance, require further study [8, 43-45].

The clinical rationale for identifying abnormalities in the reactivity of skeletal muscle vessels with NIRS is that early detection may aid risk stratification for cardiovascular events and identify women with a greater risk for type 2 diabetes and hypertension in the postpartum years. Our findings may provide a foundation for future examination of lifestyle/pharmacological interventions that will reverse this microvascular dysfunction or differentiate responders from non-responders to therapy.

It should be mentioned, however, that absolute values of $\mathrm{O}_{2} \mathrm{Hb}$ and $\mathrm{HHb}$ should be interpreted with caution if groups with different adipose tissue thickness are measured. Although in this study, groups with similar forearm skinfold thickness were compared and the blunted TSI curve was still evident in GDM vs the BMI-matched control group, emphasis in the NIRS data should be given to the dynamic adjustment of the NIRS signal, i.e. occlusion/ reperfusion slopes, as these variables are not influenced by adipose tissue thickness [46], and to the TSI signal as the normalisation of $\mathrm{O}_{2} \mathrm{Hb}$ per $\mathrm{tHb}$ in its calculation diminishes differences in signal amplitude. Otherwise, normalisation of the NIRS absolute $\mathrm{O}_{2} \mathrm{Hb}$ and $\mathrm{HHb}$ signal to adipose thickness is advised. A study limitation is the relatively small sample size. However, the strengths are that this study shows, for the first time, that despite the short duration of glucose impairment, early abnormalities in microvascular reactivity and local skeletal muscle oxygenation exist in GDM.

In conclusion, women with GDM exhibit a characteristic blunted TSI curve during an acute interruption of blood flow and a slower hyperaemic response after the ischaemic challenge compared with non-complicated pregnancies, indicating an inability of the small arterioles to dilate in response to stimuli and impairments in oxygen delivery and extraction by their skeletal muscles. This microvascular stiffening could not only limit oxygen delivery to skeletal muscles, inducing early fatigue during exercise, but possibly promote target organ damage. Alterations in microvascular function are evident in GDM before any clinical manifestations of macrovascular dysfunction or gestational hypertension. Importantly, the blunted oxygenation indices significantly correlated with early pregnancy blood glucose levels (before the GDM gravidas-initiated treatment), aortic 
stiffening indices and $24 \mathrm{~h} \mathrm{BP}$ measurement, and persisted even when hyperglycaemia was controlled.

Acknowledgements The authors would like to thank V. Sachpekidis (Papageorgiou Hospital, Thessaloniki, Greece), N. Koletsos and A.S. Zafeiridis (Aristotle University of Thessaloniki, Medical School, Thessaloniki, Greece) for their valuable assistance in data collection and patient care. The authors would also like to thank all the participants in this study.

Funding No specific funding was received for this study.

Duality of interest The authors declare that there is no duality of interest associated with this manuscript.

Contribution statement $\mathrm{KD}, \mathrm{DGG}, \mathrm{AT}, \mathrm{AZ}, \mathrm{SD}$ and EK contributed to the conception and design of the study. KD, AT, IG, EK, PP and GAT acquired the data. KD, AT, EK, PP, GAT, ISV, AZ, SD and DGG interpreted the data. $\mathrm{KD}, \mathrm{AT}$, DGG, $\mathrm{SD}, \mathrm{EK}$ and $\mathrm{AZ}$ prepared the manuscript. KD, AT, IG, EK, GAT, PP, IV, AZ, SD and DGG contributed to the discussion and reviewed/edited and approved the manuscript. KD, SD and DGG are the guarantors of this work.

\section{References}

1. American Diabetes Association (2016) Classification and diagnosis of diabetes. Diabetes Care 39(Suppl 1):S13-S22

2. Bryson CL, Ioannou GN, Rulyak SJ, Critchlow C (2003) Association between gestational diabetes and pregnancy-induced hypertension. Am J Epidemiol 158:1148-1153

3. Barden A, Singh R, Walters BN, Ritchie J, Roberman B, Beilin LJ (2004) Factors predisposing to pre-eclampsia in women with gestational diabetes. J Hypertens 22:2371-2378

4. Lee H, Jang HC, Park HK, Cho NH (2007) Early manifestation of cardiovascular disease risk factors in offspring of mothers with previous history of gestational diabetes mellitus. Diabetes Res Clin Pract 78:238-245

5. Rayanagoudar G, Hashi AA, Zamora J, Khan KS, Hitman GA, Thangaratinam S (2016) Quantification of the type 2 diabetes risk in women with gestational diabetes: a systematic review and metaanalysis of 95,750 women. Diabetologia 59:1403-1411

6. Nelson SM, Freeman DJ, Sattar N, Johnstone FD, Lindsay RS (2007) IGF-1 and leptin associate with fetal HDL cholesterol at birth: examination in offspring of mothers with type 1 diabetes. Diabetes 56:2705-2709

7. de Jager J, Dekker JM, Kooy A et al (2006) Endothelial dysfunction and low-grade inflammation explain much of the excess cardiovascular mortality in individuals with type 2 diabetes: the Hoorn Study. Arterioscler Thromb Vasc Biol 26:1086-1093

8. Bakker W, Eringa EC, Sipkema P, van Hinsbergh VW (2009) Endothelial dysfunction and diabetes: roles of hyperglycemia, impaired insulin signaling and obesity. Cell Tissue Res 335:165-189

9. Guimaraes MF, Brandao AH, Rezende CA et al (2014) Assessment of endothelial function in pregnant women with preeclampsia and gestational diabetes mellitus by flow-mediated dilation of brachial artery. Arch Gynecol Obstet 290:441-447
10. Pontes IE, Afra KF, Silva JR Jr, Borges PS, Clough GF, Alves JG (2015) Microvascular reactivity in women with gestational diabetes mellitus studied during pregnancy. Diabetol Metab Syndr 7, e27

11. Bulzico DA, Zajdenverg L, Cabizuca CA, de Oliveira JE, Salles GF (2012) Assessment of arterial stiffness in women with gestational diabetes. Diabet Med 29:227-231

12. Di Fulvio P, Pandolfi A, Formoso G et al (2014) Features of endothelial dysfunction in umbilical cord vessels of women with gestational diabetes. Nutr Metab Cardiovasc Dis 24:1337-1345

13. McLay KM, Fontana FY, Nederveen JP et al (2016) Vascular responsiveness determined by near-infrared spectroscopy measures of oxygen saturation. Exp Physiol 101:34-40

14. Sobrevia L, Abarzua F, Nien JK et al (2011) Differential placental macrovascular and microvascular endothelial dysfunction in gestational diabetes. Placenta 32(Suppl 2):S159-S164

15. Pardo F, Arroyo P, Salomon C et al (2013) Role of equilibrative adenosine transporters and adenosine receptors as modulators of the human placental endothelium in gestational diabetes mellitus. Placenta 34:1121-1127

16. McLay KM, Nederveen JP, Pogliaghi S, Paterson DH, Murias JM (2016) Repeatability of vascular responsiveness measures derived from near-infrared spectroscopy. Physiological Rep 4, e12772

17. Grassi B, Pogliaghi S, Rampichini S et al (2003) Muscle oxygenation and pulmonary gas exchange kinetics during cycling exercise on-transitions in humans. J Appl Physiol 95:149-158

18. Celie B, Boone J, Van Coster R, Bourgois J (2012) Reliability of near infrared spectroscopy (NIRS) for measuring forearm oxygenation during incremental handgrip exercise. Eur J Appl Physiol 112: 2369-2374

19. Boone J, Celie B, Dumortier J et al (2014) Forearm muscle oxygenation responses during and following arterial occlusion in patients with mitochondrial myopathy. Respir Physiol Neurobiol 190: 70-75

20. Pedersen BL, Baekgaard N, Quistorff B (2009) Muscle mitochondrial function in patients with type 2 diabetes mellitus and peripheral arterial disease: implications in vascular surgery. Eur J Vasc Endovasc Surg 38:356-364

21. Boyle KE, Hwang H, Janssen RC et al (2014) Gestational diabetes is characterized by reduced mitochondrial protein expression and altered calcium signaling proteins in skeletal muscle. PLoS One 9, e106872

22. Asmar R, Rudnichi A, Blacher J, London GM, Safar ME (2001) Pulse pressure and aortic pulse wave are markers of cardiovascular risk in hypertensive populations. Am J Hypertens 14:91-97

23. Gkaliagkousi E, Gavriilaki E, Triantafyllou A, Douma S (2015) Clinical significance of endothelial dysfunction in essential hypertension. Curr Hypertens Rep 17:e85

24. Laurent S, Cockcroft J, Van Bortel L et al (2006) Expert consensus document on arterial stiffness: methodological issues and clinical applications. Eur Heart J 27:2588-2605

25. Marin-Corral J, Claverias L, Bodi M et al (2016) Prognostic value of brachioradialis muscle oxygen saturation index and vascular occlusion test in septic shock patients. Med Intensiva/Soc Esp Med Intensiva Unidades Coronarias 40:208-215

26. Bailey SJ, Wilkerson DP, Dimenna FJ, Jones AM (2009) Influence of repeated sprint training on pulmonary $\mathrm{O} 2$ uptake and muscle deoxygenation kinetics in humans. J Appl Physiol 106:1875-1887

27. Zafeiridis A, Kounoupis A, Dipla K et al (2015) Oxygen delivery and muscle deoxygenation during continuous, long- and shortinterval exercise. Int J Sports Med 36:872-880

28. Boushel R, Langberg H, Olesen J, Gonzales-Alonzo J, Bulow J, Kjaer M (2001) Monitoring tissue oxygen availability with near infrared spectroscopy (NIRS) in health and disease. Scand J Med Sci Sports 11:213-222

29. Pepin EB, Spencer MK, Hicks RW, Jackson CG, Tran ZV (1998) Reliability of a handgrip test for evaluating heart rate and pressor 
responses in multiple sclerosis. Med Sci Sports Exerc 30:12961298

30. Bhambhani Y, Maikala R, Farag M, Rowland G (2006) Reliability of near-infrared spectroscopy measures of cerebral oxygenation and blood volume during handgrip exercise in nondisabled and traumatic brain-injured subjects. J Rehabil Res Dev 43:845-856

31. Ryan TE, Southern WM, Reynolds MA, McCully KK (2013) A cross-validation of near-infrared spectroscopy measurements of skeletal muscle oxidative capacity with phosphorus magnetic resonance spectroscopy. J Appl Physiol 115:1757-1766

32. Frijns CJ, Kappelle LJ, van Gijn J, Nieuwenhuis HK, Sixma JJ, Fijnheer R (1997) Soluble adhesion molecules reflect endothelial cell activation in ischemic stroke and in carotid atherosclerosis. Stroke 28:2214-2218

33. Joannides R, Bellien J, Thuillez C (2006) Clinical methods for the evaluation of endothelial function- a focus on resistance arteries. Fundam Clin Pharmacol 20:311-320

34. Kawano H, Motoyama T, Hirashima O et al (1999) Hyperglycemia rapidly suppresses flow-mediated endothelium-dependent vasodilation of brachial artery. J Am Coll Cardiol 34:146-154

35. Stanley JL, Sankaralingam S, Baker PN, Davidge ST (2010) Previous gestational diabetes impairs long-term endothelial function in a mouse model of complicated pregnancy. Am J Physiol Regul Integr Comp Physiol 299:R862-R870

36. Figueroa R, Omar HA, Tejani N, Wolin MS (1993) Gestational diabetes alters human placental vascular responses to changes in oxygen tension. Am J Obstet Gynecol 168:1616-1622

37. Li H, Gutterman DD, Rusch NJ, Bubolz A, Liu Y (2004) Nitration and functional loss of voltage-gated $\mathrm{K}+$ channels in rat coronary microvessels exposed to high glucose. Diabetes 53:2436-2442
38. Blacher J, Asmar R, Djane S, London GM, Safar ME (1999) Aortic pulse wave velocity as a marker of cardiovascular risk in hypertensive patients. Hypertension 33:1111-1117

39. Wedel Jones C, Mandala M, Barron C, Bernstein I, Osol G (2009) Mechanisms underlying maternal venous adaptation in pregnancy. Reprod Sci 16:596-604

40. Hall JE, do Carmo JM, da Silva AA, Wang Z, Hall ME (2015) Obesity-induced hypertension: interaction of neurohumoral and renal mechanisms. Circ Res 116:991-1006

41. Brunner S, Stecher L, Ziebarth S et al (2015) Excessive gestational weight gain prior to glucose screening and the risk of gestational diabetes: a meta-analysis. Diabetologia 58:2229-2237

42. Anastasiou E, Lekakis JP, Alevizaki M et al (1998) Impaired endothelium-dependent vasodilatation in women with previous gestational diabetes. Diabetes Care 21:2111-2115

43. Joffe GM, Esterlitz JR, Levine RJ et al (1998) The relationship between abnormal glucose tolerance and hypertensive disorders of pregnancy in healthy nulliparous women. Calcium for Preeclampsia Prevention (CPEP) Study Group. Am J Obstet Gynecol 179:10321037

44. Sibai BM, Caritis S, Hauth J et al (2000) Risks of preeclampsia and adverse neonatal outcomes among women with pregestational diabetes mellitus. National Institute of Child Health and Human Development Network of Maternal-Fetal Medicine Units. Am J Obstet Gynecol 182:364-369

45. Cruickshank K, Riste L, Anderson SG, Wright JS, Dunn G, Gosling RG (2002) Aortic pulse-wave velocity and its relationship to mortality in diabetes and glucose intolerance: an integrated index of vascular function? Circulation 106:2085-2090

46. Bopp CM, Townsend DK, Barstow TJ (2011) Characterizing nearinfrared spectroscopy responses to forearm post-occlusive reactive hyperemia in healthy subjects. Eur J Appl Physiol 111:2753-2761 